\title{
Phase I Study of Neoadjuvant Chemotherapy with Capecitabine and Oxaliplatin for Locally Advanced Gastric Cancer
}

\author{
HIRONAGA SATAKE ${ }^{1}$, MASATO KONDO ${ }^{2}$, MOTOKO MIZUMOTO ${ }^{2}$, TAKESHI KOTAKE ${ }^{1}$, \\ YOSHIHIRO OKITA ${ }^{3}$, TAKATSUGU OGATA ${ }^{1}$, YUKIMASA HATACHI ${ }^{1}$, \\ HISATERU YASUI ${ }^{1}$, AKIRA MIKI ${ }^{2}$, YUKIHIRO IMAI ${ }^{4}$, CHIHIRO ICHIKAWA $^{4}$, KENTA MUROTANI $^{5}$, \\ MASAHITO KOTAKA ${ }^{6}$, TAKESHI KATO ${ }^{7}$, SATOSHI KAIHARA ${ }^{2}$ and AKIHITO TSUJI ${ }^{3}$ \\ Departments of ${ }^{1}$ Medical Oncology, ${ }^{2}$ Surgery, and ${ }^{4}$ Pathology, \\ Kobe City Medical Center General Hospital, Kobe, Japan; \\ ${ }^{3}$ Department of Clinical Oncology, Kagawa University Hospital, Kita, Japan; \\ ${ }^{5}$ Division of Biostatistics, Clinical Research Center, Aichi Medical University, Nagakute, Japan; \\ ${ }^{6}$ Gastrointestinal Cancer Center, Sano Hospital, Kobe, Japan; \\ ${ }^{7}$ Department of Surgery, Kansai Rosai Hospital, Amagasaki, Japan
}

\begin{abstract}
Aim: To determine the recommended dose of neoadjuvant chemotherapy of combined capecitabine and oxalipatin (G-XELOX) for locally advanced gastric cancer. Patients and Methods: Patients received two cycles of neoadjuvant chemotherapy with oxaliplatin on day 1 and capecitabine $\left(2,000 \mathrm{mg} / \mathrm{m}^{2} /\right.$ day, b.i.d. $)$ on days $1-14$, repeated every 3 weeks. They then underwent gastrectomy with curative D2/3 lymph-node dissection followed by adjuvant therapy with $S-1$ for 1 year. De-escalation of oxaliplatin dose was planned (starting at level 1, oxalipatin $130 \mathrm{mg} / \mathrm{m}^{2}$ ). Results: Six patients were enrolled. The maximum tolerated dose was not reached at level 1. Oxaliplatin at $130 \mathrm{mg} / \mathrm{m}^{2}$ combined with capecitabine at $2,000 \mathrm{mg} / \mathrm{m}^{2} /$ day, b.i.d. had acceptable toxicity. No treatment-related death occurred. Most frequent drug-related adverse events during neoadjuvant G-XELOX were nausea and peripheral sensory neuropathy. One patient declined surgical resection, leaving five undergoing resection with curative intent, of whom four achieved pathological downstaging after neoadjuvant G-XELOX. Conclusion: Neoadjuvant G-XELOX was feasible in patients with locally advanced gastric cancer.
\end{abstract}

Correspondence to: Hironaga Satake, MD, Ph.D., Department of Medical Oncology, Kobe City Medical Center General Hospital, 21-1, Minatojima minamimachi, Chuo-ku, Kobe, Hyogo, 650-0047 Japan. Tel: +81 0783024321, Fax: +81 0783027537, e-mail: takeh1977@gmail.com

Key Words: Gastric cancer, neoadjuvant chemotherapy, capecitabine, xeloda, oxaliplatin, phase I.
Gastric cancer is the fifth-most frequently diagnosed malignancy and the third-leading cause of cancer death in the world (1). Surgery with lymph node dissection is the primary treatment for localized resectable gastric cancer, but more than half of such patients experience recurrence even after curative resection (2). Combined modality therapy should be considered for all patients with localized gastric cancer treated with a curative intent. However, standard treatment for locally advanced gastric cancer differs among regions, with surgery followed by adjuvant chemotherapy preferred in Asia (3), whereas adjuvant chemoradiation is more common in the US $(4,5)$. Although postoperative therapy such as adjuvant chemotherapy or chemoradiotherapy improves patient outcomes, the survival rate remains low. One possible reason is thought to be the relatively low rate of completion of preplanned adjuvant treatment $[65.8 \%$ in the ACTS-GC trial (3); $67 \%$ in the CLASSIC trial (6); and $64 \%$ in the INT0116 trial (7)].

One promising approach to improving survival in patients with locally advanced gastric cancer is preoperative chemotherapy. Preoperative chemotherapy is regarded as standard treatment in Europe and has shown safety, downstaging and improvement of $\mathrm{R} 0$ resection rate $(8,9)$. Nevertheless, prognosis remains poor in patients with clinical T4 disease, clinical T3 disease with tumor invasion into the esophagus, a special type of gastric cancer known as linitis plastica (or Borrman type 4) together with the large ulcero-invasive-type (type 3 , with a maximum diameter $\geq 8 \mathrm{~cm}$ ), and any $\mathrm{T}$ disease with bulky nodal involvement around major branched arteries to the stomach.

Oral fluoropyrimidines, capecitabine and S-1 were developed as substitutes for fluorouracil (5-FU), which is 
administered by continuous infusion via a central venous catheter. Capecitabine has been shown to be effective in the treatment of gastric cancer, and is also administered as a combination treatment with oxaliplatin. Chemotherapy combined with capecitabine plus oxaliplatin for advanced gastric cancer (G-XELOX) has shown promising efficacy with acceptable toxicity (10). To date, however, no study has described the use of neoadjuvant chemotherapy with GXELOX for locally advanced gastric cancer.

The aim of this study was to evaluate neoadjuvant chemotherapy with G-XELOX followed by D2/3 gastrectomy and adjuvant S-1 for locally advanced gastric cancer.

\section{Patients and Methods}

Eligibility criteria and patients. Eligibility criteria included the ability to take medications orally; age 20 years or older; Eastern Cooperative Oncology Group performance status (ECOG PS) score of 0 or 1; histologically proven gastric adenocarcinoma; clinical T4 disease, or clinical T3 disease in cases of tumors invading the esophagus or of the scirrhous type (type 4$)$, including large type $3(>8 \mathrm{~cm})$, and those with bulky nodal involvement around major branched arteries to the stomach on abdominal computed tomography (CT) and diagnostic laparoscopy; resectable peritoneal dissemination (pathological CY1 or P1, except for clinical CY1 or P1); adequate organ function, as defined by hemoglobin $\geq 10 \mathrm{~g} / \mathrm{dl}$, absolute neutrophil count $\geq 1,500 / \mathrm{mm}^{3}$ platelets count $\geq 100,000 / \mathrm{mm}^{3}$, total bilirubin $\leq 1.5 \mathrm{mg} / \mathrm{dl}$, serum transaminases $\leq 100 \mathrm{U} / \mathrm{l}$ and creatinine clearance $\geq 40 \mathrm{ml} / \mathrm{min}$. Exclusion criteria were evidence of chemotherapy, immunotherapy, or radiotherapy for gastric cancer; prior myocardial infarction within three months; history of unstable angina pectoris, interstitial pneumonia, fibroid lung or severe emphysema; concurrent active malignancy; uncontrolled infection; severe mental disorder; and pregnancy or lactation.

The protocol was approved by the Institutional Review Board of Kobe City Medical Center General Hospital (approval number: $\mathrm{n} 141203$ ), and the study followed the principles of the Declaration of Helsinki and Ethical Guidelines for Clinical Studies. All patients provided written informed consent for participation in this study.

Neoadjuvant and adjuvant chemotherapy. Patients received two cycles of neoadjuvant chemotherapy, consisting of capecitabine $\left(2000 \mathrm{mg} / \mathrm{m}^{2}\right.$, p.o., days $1-14$, followed by a 1 -week rest period) plus oxaliplatin at day 1 in a 3-week schedule, followed by D2 or higher surgery. Patients with pathological R0/1 resection were administered $\mathrm{S}-1\left(80 \mathrm{mg} / \mathrm{m}^{2}\right.$, p.o., days 1-28, followed by a 2 -week rest) for 1 year as adjuvant chemotherapy. The study was designed to determine the recommended dose (RD) of neoadjuvant chemotherapy. Six patients were treated at dose level $1\left(2,000 \mathrm{mg} / \mathrm{m}^{2}\right.$ capecitabine and $130 \mathrm{mg} / \mathrm{m}^{2}$ oxaliplatin). The dose of capecitabine was fixed and oxaliplatin was examined at doses of $130 \mathrm{mg} / \mathrm{m}^{2}$ (level 1), $100 \mathrm{mg} / \mathrm{m}^{2}$ (level 0) and $85 \mathrm{mg} / \mathrm{m}^{2}$ (level -1). If three or more of the six patients experienced a doselimiting toxicity (DLT), six additional patients were accrued at the next lower dose level. The maximum tolerated dose (MTD) was defined as the dose that induced DLT during the neoadjuvant chemotherapy period in $50 \%$ or more of the participants. The RD was defined as one dose level below the MTD. If the MTD was not achieved even at level 1 , it was regarded as the RD. DLT was defined as any of the following adverse events occurring in the neoadjuvant chemotherapy period: (i) grade 4 neutropenia lasting $>4$ days; (ii) grade 4 thrombocytopenia $\left(<25,000 / \mathrm{mm}^{3}\right)$; (iii) febrile neutropenia; (iv) grade 3 or 4 nonhematological toxic effects; (v) a treatment delay longer than 14 days due to drug-related toxicity in the neoadjuvant chemotherapy periods; or (vi) treatment-related death. Neoadjuvant chemotherapy was administered tri-weekly until disease progression, unacceptable toxicity, or withdrawal of consent for up to two cycles. The dose was modified for each patient based on hematologic or non-hematologic toxicity. Neoadjuvant chemotherapy was delayed if, on the planned day of treatment, neutrophils were less than $1,500 / \mathrm{mm}^{3}$, platelets were less than $75,000 / \mathrm{mm}^{3}$, or total bilirubin was more than $2.0 \mathrm{mg} / \mathrm{dl}$; or if persistent diarrhea, mucositis higher than grade 1, or peripheral sensory neurotoxicity higher than grade 2 was present. In patients with pharyngolaryngeal dysesthesia, the duration of oxaliplatin infusion was prolonged from 2 to $6 \mathrm{~h}$. In the event of grade 4 non-hematologic toxicities, treatment was definitively interrupted.

Surgery. Tumor resectability was assessed after completion of neoadjuvant chemotherapy. Resection criteria were (i) R0 resection was anticipated by D2 or extended D2 gastrectomy; (ii) sufficient organ function; and (iii) no active infection. Patients who fulfilled these criteria were subjected to surgery within 6 weeks of the last administration of neoadjuvant chemotherapy. Combined resection of adjacent organs was permitted when these procedure were indispensable for curative resection.

Study assessment. Pretreatment evaluation included a medical history; physical examination; complete blood cell count and serum chemistry tests; esophagogastroduodenoscopy; and chest, abdominal and pelvic CT scans. Clinical examination and biochemical tests were required before and during each cycle. All adverse events during chemotherapy were evaluated by the National Cancer Institute Common Terminology Criteria for Adverse Events (CTCAE version 4.0) (11). All adverse events related to surgery were evaluated by both CTCAE version 4.0 and the Clavien-Dindo classification (12).

We planned to assess quality of life (QoL) every 3 weeks from the date of the first administration of neoadjuvant chemotherapy (baseline) to the date of surgery; and then following surgery, every 3 months from the date of the first administration of adjuvant chemotherapy for 1 year. QoL assessment was carried using the Japanese version of the Functional Assessment of Cancer TherapyGastric instrument (FACT-Ga) (13) and Functional Assessment of Cancer Therapy-Neurotoxicity (FACT-GOG-Ntx) (14). The FACT-Ga is a 46-item questionnaire that measures both general health-related quality of life (HRQOL) (FACT-G) and an additional HRQOL specifically related to a gastric cancer (GaCS) subscale. The FACTGOG-Ntx is a 38 -item questionnaire comprising two components, FACT-G and an 11-item neurotoxicity (Ntx) subscale. The range of possible score is from 0 to 184 for FACT-Ga and from 0 to 152 for FACT-GOG-Ntx score, with higher scores indicating better HRQOL. The questionnaires were distributed to the patients by the clinical research coordinators, and were completed by each patient independently.

Surgical specimens were evaluated pathologically and each case was evaluated independently by two histopathologists (Y.I and C.I). Pathological response was scored using Becker criteria (15) and Japanese criteria $(16,17)$. Surgical specimens were pathologically evaluated and graded according to the proportion of the tumor 
affected by degeneration or necrosis. The Becker criteria were graded as follows: Grade 1, complete ( $0 \%$ residual tumor; grade 1a) or subtotal tumor regression $(<10 \%$ residual tumor per tumor bed; grade $1 \mathrm{~b}$ ); grade 2, partial tumor regression (10-50\% residual tumor per tumor bed); and grade 3 , minimal or no tumor regression $(>50 \%$ residual tumor per tumor bed). The Japanese criteria were graded as follows: Grade 0 , none of the tumor affected; grade $1 \mathrm{a},<1 / 3$ affected; grade $1 b, \geq 1 / 3$ and $<2 / 3$ affected; grade $2, \geq 2 / 3$ affected; and grade 3 , no residual tumor. Peritoneal lavage cytology (CY) was diagnosed from either ascites or peritoneal lavage and was classified as CY1 (positive) and CY0 (negative).

End-points. The primary endpoints in this study were the MTD and RD of this neoadjuvant G-XELOX regimen. Secondary endpoints included pathological response rate ( $\mathrm{pRR}$ ), relapse-free survival (RFS), overall survival (OS), curative resection rate (R0, R0/1 resection rate), relative dose intensity (RDI), completion rate of neoadjuvant/adjuvant chemotherapy and safety. Dose intensity was calculated as the ratio of the actual to planned dose intensity in milligrams per square meter per week. Safety and efficacy analyses were both conducted on an intention-to-treat population, defined as all patients enrolled in the study who received at least one dose of chemotherapy. The RFS was defined as the time from the date of the first administration of neoadjuvant chemotherapy to the first documentation of disease relapse or death. OS was determined from the date of the first administration of neoadjuvant chemotherapy to the date of death or last confirmation of survival. Statistical data were obtained using the SPSS software package (SPSS 22.0 Inc.; Chicago, IL, USA).

This trial was registered with University Hospital Medical Information Network (no. UMIN000015950).

\section{Results}

Patients. From February to October 2015, six patients were enrolled. Characteristics of the enrolled patients are listed in Table I. All patients were chemo-naïve and had a good PS. All cases were clinical T4a stage and two cases of clinical stage IV were pathological CY1, P0 and CY0, P1, respectively, as confirmed by pre-treatment diagnostic laparoscopy.

Neoadjuvant chemotherapy and toxicities. Six patients were enrolled at dose level $1 \quad\left(2,000 \mathrm{mg} / \mathrm{m}^{2} /\right.$ day capecitabine, $130 \mathrm{mg} / \mathrm{m}^{2}$ oxaliplatin). No patient had a DLT at level 1, and hence the RD was determined to be $2,000 \mathrm{mg} / \mathrm{m}^{2} /$ day capecitabine, and $130 \mathrm{mg} / \mathrm{m}^{2}$ oxaliplatin for phase II study.

The worst toxicity through the neoadjuvant chemotherapy period is listed in Table II. All patients completed the two cycles of neoadjuvant chemotherapy, and toxicity was assessable in all patients. No grade 3 or higher toxicities occurred. Peripheral neuropathy was observed in all patients but with no functional disorders. No treatment-related death was observed. No patient discontinued neoadjuvant GXELOX, although the second cycle was delayed in one patient due to a national holiday. Furthermore, two patients required dose reduction in both oxaliplatin (due to grade 2
Table I. Patient characteristics $(n=6)$.

\begin{tabular}{|c|c|c|c|}
\hline \multicolumn{4}{|l|}{ Variable } \\
\hline Age, years & Median (range) & 72 & $(68-79)$ \\
\hline \multirow[t]{2}{*}{ Gender, $\mathrm{n}(\%)$} & Male & 5 & 83 \\
\hline & Female & 1 & 17 \\
\hline \multirow[t]{2}{*}{ ECOG PS, n (\%) } & 0 & 6 & 100 \\
\hline & 1 & 0 & 0 \\
\hline \multirow[t]{3}{*}{ Primary tumor location, n (\%) } & $\mathrm{U}$ & 1 & 17 \\
\hline & $\mathrm{M}$ & 1 & 17 \\
\hline & $\mathrm{L}$ & 4 & 67 \\
\hline \multirow[t]{2}{*}{ Histology, n (\%) } & Intestinal & 3 & 50 \\
\hline & Diffuse & 3 & 50 \\
\hline \multirow[t]{2}{*}{ Clinical T-stage, n (\%) } & $\mathrm{T} 3$ & 0 & 0 \\
\hline & $\mathrm{T} 4$ & 6 & 100 \\
\hline \multirow[t]{4}{*}{ Clinical N-stage, n (\%) } & No & 1 & 17 \\
\hline & N1 & 1 & 17 \\
\hline & N2 & 4 & 67 \\
\hline & $\mathrm{N} 3$ & 0 & 0 \\
\hline \multirow[t]{6}{*}{ TNM stage, $\mathrm{n}(\%)$} & IIA & 0 & 0 \\
\hline & IIB & 1 & 17 \\
\hline & IIIA & 1 & 17 \\
\hline & IIIB & 2 & 33 \\
\hline & IIIC & 0 & 0 \\
\hline & IV & 2 & 33 \\
\hline \multirow[t]{4}{*}{ Macroscopic type, n (\%) } & 1 & 0 & 0 \\
\hline & 2 & 3 & 50 \\
\hline & 3 & 2 & 33 \\
\hline & 4 & 1 & 17 \\
\hline
\end{tabular}

ECOG PS, Eastern Cooperative Oncology Group performance status; $\mathrm{U}$ : upper third; M: middle third; L: lower third.

thrombocytopenia in the first patient and grade 2 fatigue in the second) and capecitabine (due to grade 2 nausea). The average percentage of dose intensity delivered during neoadjuvant chemotherapy was $75.6 \%$ for capecitabine and $95.9 \%$ for oxaliplatin.

Surgery. After one patient refused surgical resection, five out of the six patients who completed neoadjuvant chemotherapy underwent surgery. The median time from start of neoadjuvant G-XELOX to surgery was 59 days (range=5382 days), and median time from its completion was 28 days (range=16-37 days). Surgical findings are shown in Table III. Resection with curative intent (R0) was undertaken in all five patients.

There was no operative mortality. Operative morbidity was observed in all five patients including pancreatic fistula (one case was grade 2 in CTCAE version 4.0 and grade IIIa in the Clavien-Dindo classification, one case was grade 1 in both CTCAE version 4.0 and the Clavien-Dindo classification, and one case was grade 2 in both CTCAE version 4.0 and the Clavien-Dindo classification), abdominal abscess (grade 3 in CTCAE version 4.0 and grade II in the 
Table II. Maximum toxicity per patient during neoadjuvant chemotherapy ( $n=6)$.

\begin{tabular}{|c|c|c|c|c|c|c|}
\hline \multirow[t]{2}{*}{ Adverse event } & \multicolumn{5}{|c|}{ NCI-CTC grade } & \multirow[b]{2}{*}{ Grade $3 / 4, \%$} \\
\hline & $1, \mathrm{n}$ & $2, \mathrm{n}$ & $3, \mathrm{n}$ & $4, \mathrm{n}$ & All, $\%$ & \\
\hline HematologicalLeukopenia & 1 & 1 & 0 & 0 & 33 & 0 \\
\hline Neutropenia & 0 & 2 & 0 & 0 & 33 & 0 \\
\hline Anemia & 3 & 0 & 0 & 0 & 50 & 0 \\
\hline Thrombocytopenia & 2 & 1 & 0 & 0 & 50 & 0 \\
\hline Creatinine increased & 2 & 0 & 0 & 0 & 33 & 0 \\
\hline \multicolumn{7}{|l|}{ Non-hematological } \\
\hline Anorexia & 3 & 0 & 0 & 0 & 50 & 0 \\
\hline Constipation & 2 & 0 & 0 & 0 & 33 & 0 \\
\hline Diarrhea & 0 & 1 & 0 & 0 & 17 & 0 \\
\hline Dysgeusia & 1 & 0 & 0 & 0 & 17 & 0 \\
\hline Fatigue & 0 & 1 & 0 & 0 & 17 & 0 \\
\hline Febrile neutropenia & - & - & 0 & 0 & 0 & 0 \\
\hline Hand-foot syndrome & 2 & 0 & 0 & 0 & 33 & 0 \\
\hline Hiccups & 3 & 0 & 0 & 0 & 50 & 0 \\
\hline Hoarseness & 1 & 0 & 0 & 0 & 17 & 0 \\
\hline Mucositis & 2 & 0 & 0 & 0 & 33 & 0 \\
\hline Nausea & 2 & 2 & 0 & 0 & 67 & 0 \\
\hline Peripheral sensory neuropathy & 6 & 0 & 0 & 0 & 100 & 0 \\
\hline Vomiting & 1 & 0 & 0 & 0 & 17 & 0 \\
\hline
\end{tabular}

NCI-CTC, National Cancer Institute Common Toxicity Criteria.

Clavien-Dindo classification), leakage (grade 3 in CTCAE version 4.0 and grade IIIa in the Clavien-Dindo classification) and surgical site infection (grade 1 in both CTCAE version 4.0 and the Clavien-Dindo classification). One patient had both pancreatic fistula (grade 2 in both CTCAE version 4.0 and the Clavien-Dindo classification) and leakage. No patients required re-operation for morbidity. Median duration of hospitalization after operation was 17 days (range $=8-70$ ).

Pathological response. Table IV shows the pathological findings in all resected patients. One specimen had Grade 2 regression (10-50\% residual tumor), and four specimens had more than $50 \%$ remaining residual tumor, corresponding to Grade 3 in the Becker criteria. In the Japanese criteria, one specimen was Grade $1 \mathrm{~b}(\geq 1 / 3$ and $<2 / 3$ affected) and four were Grade 1a $(<1 / 3$ affected). From these results, the pathological complete response (pCR) rate was $0 \%$ and the pathological response rate (pRR) was $20 \%$ according to the Becker criteria and Japan criteria. Four patients $(80 \%)$ achieved a pathological downstaging after neoadjuvant GXELOX therapy.

Adjuvant chemotherapy and toxicities. Of the six patients, one patient declined surgical resection, leaving five patients to receive adjuvant chemotherapy. Median time from surgery
Table III. Surgical findings and complications $(\mathrm{n}=5)$.

\begin{tabular}{llc}
\hline & & Value \\
\hline Type of surgery & Total gastrectomy, n (\%) & $1(20)$ \\
& Distal gastrectomy, n (\%) & $4(80)$ \\
Dissection & D2, n (\%) & $5(100)$ \\
Combined resection & Gall bladder, n (\%) & $4(80)$ \\
& Small intestine, n (\%) & $1(20)$ \\
Peritoneal cytology & Negative, n (\%) & $5(100)$ \\
& Positive, n (\%) & $0(0)$ \\
Operation time, min & Mean (range) & $299(246-339)$ \\
Blood loss, ml & Mean (range) & $529(146-1056)$ \\
Complications & Pancreatic fistula, n (\%) & $3(60)^{*}$ \\
& Abdominal abscess, n (\%) & $1(20)$ \\
& Anastomotic leakage, n (\%) & $1(20)^{*}$ \\
& Surgical site infection, n (\%) & $1(20)$ \\
\hline
\end{tabular}

*One patient had both pancreatic fistula and leakage.

to the start of adjuvant chemotherapy was 41 days (range=23-74). The safety of adjuvant S-1 monotherapy was assessed in all five patients. The worst toxicities associated with adjuvant chemotherapy with $\mathrm{S}-1$ are summarized in Table V. Of the five patients, three completed adjuvant S-1 monotherapy while two discontinued, one due to prolonged thrombocytopenia even with a dose reduction and one due 
Table IV. Pathological findings in all patients who underwent resection ( $n=5)$.

\begin{tabular}{lccccccccccc}
\hline Case & Lauren & $\mathrm{cT}$ & $\mathrm{cN}$ & $\mathrm{cM}$ & $\mathrm{cStage}$ & $\mathrm{ypT}$ & $\mathrm{ypN}$ & $\mathrm{yM}$ & ypStage & JGCA criteria & Becker criteria \\
\hline 1 & Intestinal & $4 \mathrm{a}$ & 2 & 0 & IIIB & 2 & 1 & 0 & IIA & $1 \mathrm{a}$ & 3 \\
2 & Diffuse & $4 \mathrm{a}$ & 2 & 0 & IIIB & $4 \mathrm{a}$ & 2 & 0 & IIIB & $1 \mathrm{a}$ & 3 \\
3 & Diffuse & $4 \mathrm{a}$ & 2 & 1 & IV & $4 \mathrm{a}$ & $3 \mathrm{~b}$ & 0 & IIIC & $1 \mathrm{a}$ & 3 \\
4 & Intestinal & $4 \mathrm{a}$ & 1 & 0 & IIIA & 2 & 1 & 0 & IIA & $1 \mathrm{a}$ & 3 \\
5 & Diffuse & $4 \mathrm{a}$ & 2 & 1 & IV & $4 \mathrm{a}$ & $3 \mathrm{a}$ & 0 & IIIC & $1 \mathrm{~b}$ & 2 \\
\hline
\end{tabular}

JGCA, Japanese Gastric Cancer Association.

Table V. Maximum toxicity per patient during adjuvant $S-1$ monotherapy $(n=5)$.

\begin{tabular}{|c|c|c|c|c|c|c|}
\hline \multirow[t]{2}{*}{ Adverse event } & \multicolumn{5}{|c|}{ NCI CTC grade } & \multirow[b]{2}{*}{ Grade $3 / 4, \%$} \\
\hline & $1, \mathrm{n}$ & $2, \mathrm{n}$ & $3, \mathrm{n}$ & $4, n$ & All, $\%$ & \\
\hline \multicolumn{7}{|l|}{ Hematological } \\
\hline Leukopenia & 1 & 1 & 1 & 0 & 60 & 20 \\
\hline Neutropenia & 0 & 0 & 2 & 0 & 40 & 40 \\
\hline Anemia & 3 & 2 & 0 & 0 & 100 & 0 \\
\hline Thrombocytopenia & 3 & 0 & 0 & 0 & 60 & 0 \\
\hline Creatinine increased & 1 & 0 & 0 & 0 & 20 & 0 \\
\hline \multicolumn{7}{|l|}{ Non-hematological } \\
\hline Anorexia & 2 & 0 & 0 & 0 & 40 & 0 \\
\hline Diarrhea & 2 & 1 & 0 & 0 & 60 & 0 \\
\hline Dizziness & 1 & 0 & 0 & 0 & 20 & 0 \\
\hline Dysgeusia & 1 & 0 & 0 & 0 & 20 & 0 \\
\hline Fatigue & 1 & 0 & 0 & 0 & 20 & 0 \\
\hline Febrile neutropenia & - & - & 0 & 0 & 0 & 0 \\
\hline Hand-foot syndrome & 1 & 0 & 0 & 0 & 20 & 0 \\
\hline Hypertension & 0 & 1 & 0 & 0 & 20 & 0 \\
\hline Nausea & 2 & 0 & 0 & 0 & 40 & 0 \\
\hline Peripheral sensory neuropathy & 2 & 0 & 0 & 0 & 40 & 0 \\
\hline Rash maculo-papular & 1 & 0 & 0 & 0 & 20 & 0 \\
\hline Vomiting & 1 & 0 & 0 & 0 & 20 & 0 \\
\hline
\end{tabular}

NCI CTC, National Cancer Institute Common Toxicity Criteria.

to recurrence. Average relative dose intensity was $75.1 \%$ (range $=58.1-98.4 \%)$.

Quality of life. All patients were assessed for QoL using FACT-Ga and FACT/GOG-Ntx version 4. The time course of mean FACT-Ga and FACT-GOG-Ntx scores are shown in Figure 1. QoL deteriorated due to sensory neuropathy immediately after the initiation of neoadjuvant G-XELOX, but subsequently recovered to approximately the previous level after the neoadjuvant chemotherapy and surgery.

Survival. With a median follow-up period of 24.6 months (range=16.8-27.3 months), no patient has died but one has experienced liver metastasis.

\section{Discussion}

In this study, we evaluated the DLTs of neoadjuvant chemotherapy with capecitabine plus oxaliplatin (GXELOX) and determined the MTD and RD for locally advanced gastric cancer. RDs of neoadjuvant chemotherapy were defined as $130 \mathrm{mg} / \mathrm{m}^{2}$ oxaliplatin in combination with $2,000 \mathrm{mg} / \mathrm{m}^{2} /$ day capecitabine, b.i.d. Activity and safety with this regimen were sufficient to warrant phase II testing. To our knowledge, this is the first report of the feasibility and activity of neoadjuvant chemotherapy in patients with locally advanced gastric cancer.

Complete surgical resection provides the only chance for cure in gastric cancer. Unfortunately, many patients 


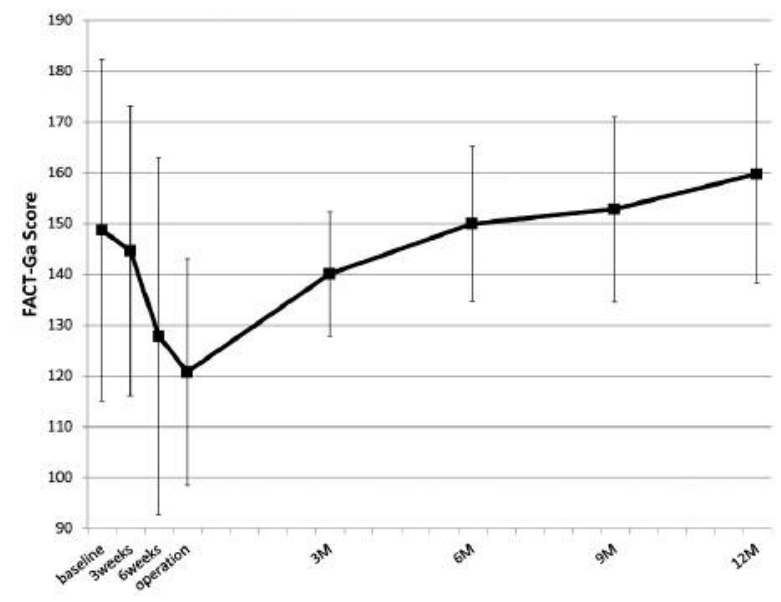

Figure 1. Time course of Functional Assessment of Cancer TherapyGastric instrument quality of life (FACT-Ga QoL) scale after initiation of neoadjuvant capecitabine plus oxaliplatin therapy.

experience recurrence, particularly in cases of T4 tumors, bulky nodal metastases, and large tumors of type 3 and 4 . The pattern of recurrence not only includes locoregional relapse but also peritoneal metastass and hematogenous metastasis. Systemic treatment strategy should, therefore, consist of multimodal treatment for resectable gastric cancer.

Allowing for the small number of patients, this study suggests that the safety of neoadjuvant G-XELOX is promising. Grade 3 or higher toxicities did not occur throughout the preoperative period, and all consenting patients were able to be treated by radical surgery, with a median time from the start of neoadjuvant G-XELOX to surgery of 59 days. Gastrectomy is generally performed 3 to 6 weeks after completion of the last cycle of chemotherapy. This recommendation is based on empirical evidence from the MAGIC and the French FNCLCC/FFCD trials, in which surgery was performed 3 to 6 weeks and 4 to 6 weeks after the completion of neoadjuvant chemotherapy, respectively $(8,9)$. It is unclear whether delayed gastrectomy compromises patient outcomes. However, in our present study, all consenting patients were able to undergo gastrectomy a median of 28 days (range $=16-37$ days) after the completion of neoadjuvant G-XELOX. This suggests that two cycles of this neoadjuvant G-XELOX regimen are safe.

Although the pathological response rate was insufficient $(20 \%)$, the pathological down-staging rate appeared attractive (80\%). Furthermore, both cases of clinical stage IV disease with pathological CY1 and P1 confirmed by diagnostic laparoscopy displayed negative peritoneal cytology after two cycles of neoadjuvant G-XELOX. Allowing for the small number of patients, these results appear to support the efficacy of the G-XELOX regimen in the treatment of gastric cancer.

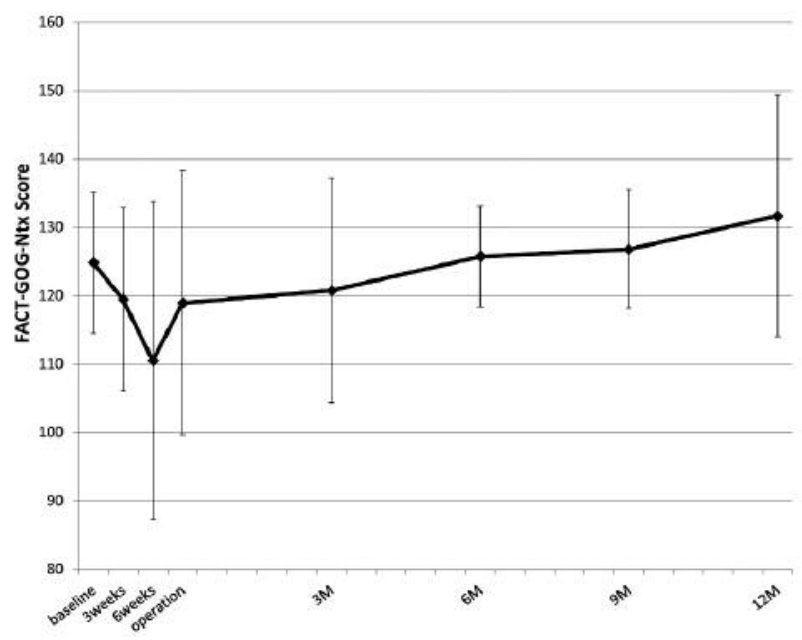

Figure 2. Time course of Functional Assessment of Cancer TherapyNeurotoxicity quality of life (FACT/GOG-NTX QoL) scale after initiation of neoadjuvant capecitabine plus oxaliplatin therapy.

Oxaliplatin is associated with a commonly occurring acute and chronic neuropathy, and oxaliplatin-induced neurotoxicity may continue after the chemotherapy and interfere with patients' daily activities. In this study, we planned two cycles of oxaliplatin administration and evaluated oxaliplatin-induced neuropathy using FACT-Ga and FACT/GOG-Ntx assessment. QoL deteriorated due to sensory neuropathy immediately after the initiation of neoadjuvant G-XELOX, but subsequently recovered to approximately the previous level after neoadjuvant chemotherapy and surgery.

Several limitations of this study related to study design should be discussed. We initially planned a de-escalation design starting with oxaliplatin at $130 \mathrm{mg} / \mathrm{m}^{2}$, which is regarded as a global standard dose $(6,10,18)$. The dose of oxaliplatin did not reach the MTD, however, and the question therefore remains whether the oxaliplatin dose could have been escalated in patients with locally advanced gastric cancer at the preoperative setting. Furthermore, the optimum number of neoadjuvant chemotherapy administrations remains to be established. The COMPASS trial, which compared neoadjuvant chemotherapy with two and four cycles of S-1 plus cisplatin and paclitaxel and cisplatin using a two-by-two factorial design for locally advanced gastric cancer, showed that the four-cycle neoadjuvant chemotherapy did not achieve a statistical improvement in survival in comparison to the two-cycle treatment, and concluded that two cycles of neoadjuvant chemotherapy should be recommended (19). Several phase II trials with XELOX in patients with advanced gastric cancer using the same treatment schedule as our study $\left(2,000 \mathrm{mg} / \mathrm{m}^{2} /\right.$ day capecitabine on days $1-14,130 \mathrm{mg} / \mathrm{m}^{2}$ oxaliplatin on day 1 , 
repeated every 3 weeks) have shown tolerable toxicity profiles (neutropenia, 6-13\%; thrombocytopenia, 6-11.3\%) $(18,20,21,22)$. However, the CLASSIC trial (6) using the same treatment schedule of G-XELOX in the adjuvant setting reported toxicities of grade 3 or higher in $56 \%$ of patients, withdrawal of consent due to toxicity in $10 \%$ and dose modifications in $90 \%$. Relatively low toxicity is required for neoadjuvant chemotherapy because target tumors are resectable or marginally resectable and the patients must undergo potentially curative surgery after chemotherapy. Furthermore, our previous phase I study of two courses (6 weeks) of neoadjuvant chemotherapy with S1 and oxaliplatin showed a pathological complete response rate of $33 \%$ (23). We therefore planned this study with two courses ( 6 weeks) of neoadjuvant chemotherapy, and toxicity was acceptable.

In conclusion, this phase I study demonstrated that RDs of neoadjuvant chemotherapy with capecitabine plus oxaliplatin was $130 \mathrm{mg} / \mathrm{m}^{2}$ oxaliplatin in combination with $2,000 \mathrm{mg} / \mathrm{m}^{2} /$ day capecitabine, b.i.d.. This regimen demonstrated sufficient activity to warrant phase II testing.

\section{Funding}

None.

\section{Conflicts of Interest}

Hironaga Satake has received honoraria from Bayer, Chugai Pharma, Eli Lilly Japan, Merck Serono, Takeda, Taiho Pharmaceutical, and Yakult Honsha. Akihito Tsuji has received honoraria from Chugai Pharma, Takeda Pharmaceutical, Eli-Lilly, Taiho Pharma, Bayer, Bristol-Myers Squibb Japan, Daiichi Sankyo and Merck Serono. Masahito Kotaka has received honoraria from Chugai Pharma, Takeda Pharmaceutical, Yakult, Taiho Pharma and Merck Serono. Takeshi Kato has received honoraria and/or research funding from Chugai Pharma, Takeda Pharmaceutical, Eli-Lilly, Taiho Pharma, Bayer and Merck Serono. All remaining Authors have declared no conflict of interest.

\section{Acknowledgements}

The Authors would like to thank the patients and families who participated in this study. The Authors also thank Rie Tamaki, Saori Tokuhara, Chiori Taniguchi and Mizuho Kouno for their support. The study material was presented in part at the 2017 Gastrointestinal Cancers Symposium, San Francisco, January 19-21, 2017.

\section{References}

1 Ferlay J, Soerjomataram I, Dikshit R, Eser S, Mathers C, Rebelo M, Parkin DM, Forman D and Bray F: Cancer incidence and mortality worldwide: sources, methods and major patterns in GLOBOCAN 2012. Int J Cancer 136: E359-386, 2015.

2 Gallo A and Cha C: Updates on esophageal and gastric cancers. World J Gastroenterol 12: 3237-3242, 2006.
3 Sakuramoto S, Sasako M, Yamaguchi T, Kinoshita T, Fujii M, Nashimoto A, Furukawa H, Nakajima T, Ohashi Y, Imamura H, Higashino M, Yamamura Y, Kurita A, Arai K and Group A-G: Adjuvant chemotherapy for gastric cancer with S-1, an oral fluoropyrimidine. N Engl J Med 357: 1810-1820, 2007.

4 Macdonald JS, Smalley SR, Benedetti J, Hundahl SA, Estes NC, Stemmermann GN, Haller DG, Ajani JA, Gunderson LL, Jessup JM and Martenson JA: Chemoradiotherapy after surgery compared with surgery alone for adenocarcinoma of the stomach or gastroesophageal junction. N Engl J Med 345: 725-730, 2001.

5 Smalley SR, Benedetti JK, Haller DG, Hundahl SA, Estes NC, Ajani JA, Gunderson LL, Goldman B, Martenson JA, Jessup JM, Stemmermann GN, Blanke CD and Macdonald JS: Updated analysis of SWOG-directed intergroup study 0116: a phase III trial of adjuvant radiochemotherapy versus observation after curative gastric cancer resection. J Clin Oncol 30: 2327-2333, 2012.

6 Bang YJ, Kim YW, Yang HK, Chung HC, Park YK, Lee KH, Lee KW, Kim YH, Noh SI, Cho JY, Mok YJ, Kim YH, Ji J, Yeh TS, Button P, Sirzen F, Noh SH and investigators Ct: Adjuvant capecitabine and oxaliplatin for gastric cancer after D2 gastrectomy (CLASSIC): a phase 3 open-label, randomised controlled trial. Lancet 379: 315-321, 2012.

7 Macdonald JS, Smalley SR, Benedetti J, Hundahl SA, Estes NC, Stemmermann GN, Haller DG, Ajani JA, Gunderson LL, Jessup JM and Martenson JA: Chemoradiotherapy after surgery compared with surgery alone for adenocarcinoma of the stomach or gastroesophageal junction. N Engl J Med 345: 725$730,2001$.

8 Cunningham D, Allum WH, Stenning SP, Thompson JN, Van de Velde CJ, Nicolson M, Scarffe JH, Lofts FJ, Falk SJ, Iveson TJ, Smith DB, Langley RE, Verma M, Weeden S, Chua YJ and Participants MT: Perioperative chemotherapy versus surgery alone for resectable gastroesophageal cancer. N Engl J Med 355: 11-20, 2006.

9 Ychou M, Boige V, Pignon JP, Conroy T, Bouche O, Lebreton G, Ducourtieux M, Bedenne L, Fabre JM, Saint-Aubert B, Geneve J, Lasser P and Rougier P: Perioperative chemotherapy compared with surgery alone for resectable gastroesophageal adenocarcinoma: an FNCLCC and FFCD multicenter phase III trial. J Clin Oncol 29: 1715-1721, 2011.

10 Kim GM, Jeung HC, Rha SY, Kim HS, Jung I, Nam BH, Lee $\mathrm{KH}$ and Chung HC: A randomized phase II trial of S-1oxaliplatin versus capecitabine-oxaliplatin in advanced gastric cancer. Eur J Cancer 48: 518-526, 2012.

11 National Institutes of Health National Cancer Institute: Common Terminology Criteria for Adverse Events (CTCAE) Version 4.0. U.S. DEPARTMENT OF HEALTH AND HUMANS SERVICES Published: May 28, 2009 (v4.03: June 14, 2010).

12 Clavien PA, Barkun J, de Oliveira ML, Vauthey JN, Dindo D, Schulick RD, de Santibanes E, Pekolj J, Slankamenac K, Bassi C, Graf R, Vonlanthen R, Padbury R, Cameron JL and Makuuchi M: The Clavien-Dindo classification of surgical complications: five-year experience. Ann Surg 250: 187-196, 2009.

13 Garland SN, Pelletier G, Lawe A, Biagioni BJ, Easaw J, Eliasziw M, Cella D and Bathe OF: Prospective evaluation of the reliability, validity and minimally important difference of the functional assessment of cancer therapy-gastric (FACT-Ga) quality-of-life instrument. Cancer 117: 1302-1312, 2011. 
14 Calhoun EA, Welshman EE, Chang CH, Lurain JR, Fishman DA, Hunt TL and Cella D: Psychometric evaluation of the Functional Assessment of Cancer Therapy/Gynecologic Oncology Group-Neurotoxicity (Fact/GOG-Ntx) questionnaire for patients receiving systemic chemotherapy. Int J Gynecol Cancer 13: 741-748, 2003.

15 Becker K, Mueller JD, Schulmacher C, Ott K, Fink U, Busch R, Bottcher K, Siewert JR and Hofler H: Histomorphology and grading of regression in gastric carcinoma treated with neoadjuvant chemotherapy. Cancer 98: 1521-1530, 2003.

16 Association JGC: Japanese Classification of Gastric Carcinoma, 14th Edition. Tokyo: Kanehara, Inc, 2010.

17 Ninomiya Y, Yanagisawa A, Kato Y, Kitagawa T, Ishihara S and Nakajima T: Histological indications of a favorable prognosis with far-advanced gastric carcinomas after preoperative chemotherapy. J Cancer Res Clin Oncol 125: 699-706, 1999.

18 Park YH, Lee JL, Ryoo BY, Ryu MH, Yang SH, Kim BS, Shin DB, Chang HM, Kim TW, Yuh YJ and Kang YK: Capecitabine in combination with Oxaliplatin (XELOX) as a first-line therapy for advanced gastric cancer. Cancer Chemother Pharmacol 61: 623-629, 2008.

19 Yoshikawa T, Morita S, Tanabe K, Nishikawa K, Ito Y, Matsui T, Fujitani K, Kimura Y, Fujita J, Aoyama T, Hayashi T, Cho H, Tsuburaya A, Miyashita Y and Sakamoto J: Survival results of a randomised two-by-two factorial phase II trial comparing neoadjuvant chemotherapy with two and four courses of $\mathrm{S}-1$ plus cisplatin (SC) and paclitaxel plus cisplatin (PC) followed by D2 gastrectomy for resectable advanced gastric cancer. Eur J Cancer 62: 103-111, 2016.
20 Dong N, Jiang W, Li H, Liu Z, Xu X and Wang M: Triweekly oxaliplatin plus oral capecitabine as first-line chemotherapy in elderly patients with advanced gastric cancer. Am J Clin Oncol 32: 559-563, 2009.

21 Liu C, Sun Q, Hang X, Zhong B and Wang D: Multicenter phase II study of capecitabine plus oxaliplatin as a first-line therapy in Chinese patients with advanced gastric cancer. Anticancer Drugs 19: 825-831, 2008.

22 Luo HY, Xu RH, Wang F, Qiu MZ, Li YH, Li FH, Zhou ZW and Chen XQ: Phase II trial of XELOX as first-line treatment for patients with advanced gastric cancer. Chemotherapy 56: 94-100, 2010.

23 Satake H, Miki A, Kondo M, Kotake T, Okita Y, Hatachi Y, Yasui $\mathrm{H}$, Imai $\mathrm{Y}$, Ichikawa $\mathrm{C}$, Murotani $\mathrm{K}$, Hashida $\mathrm{H}$, Kobayashi H, Kotaka M, Kato T, Kaihara S and Tsuji A: Phase I study of neoadjuvant chemotherapy with S-1 and oxaliplatin for locally advanced gastric cancer (Neo G-SOX PI). ESMO Open 2: e000130, 2017. 